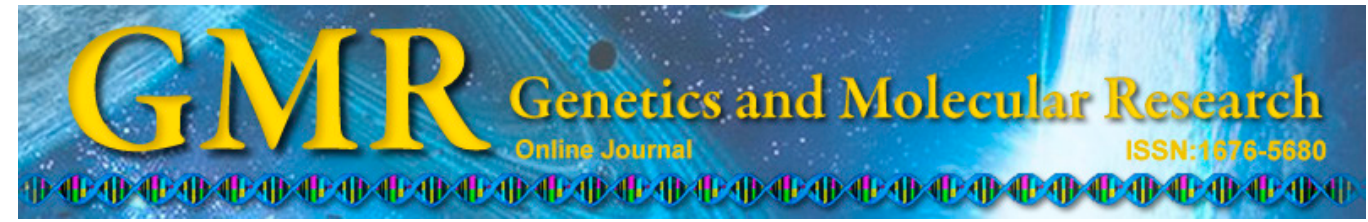

\title{
Association between IL-21 polymorphism and systemic lupus erythematosus: a meta-analysis
}

\author{
J.H. Qi ${ }^{1}$, J. Qi ${ }^{2}$, L.N. Xiang' ${ }^{1}$ and G. Nie $^{3}$ \\ ${ }^{1}$ Department of Hubei Key Laboratory of Kidney Disease Pathogenesis and \\ Intervention, Huangshi Central Hospital, \\ Affiliated Hospital of Hubei Polytechnic University, Huangshi, China \\ ${ }^{2}$ Department of Burns, People's Hospital of Lanling County, Lanling, China \\ ${ }^{3}$ Department of Dermatology, Huangshi Central Hospital, \\ Affiliated Hospital of Hubei Polytechnic University, Huangshi, China \\ Corresponding author: G. Nie \\ E-mail: niegang2014@126.com
}

Genet. Mol. Res. 14 (3): 9595-9603 (2015)

Received December 12, 2014

Accepted May 8, 2015

Published August 14, 2015

DOI http://dx.doi.org/10.4238/2015.August.14.22

\begin{abstract}
Several case-control studies have been conducted to investigate the association between Interleukin-21 (IL-21) polymorphisms and systemic lupus erythematosus (SLE) susceptibility, and most of the studies focused on $I L-21$ rs907715 and rs2221903 polymorphisms. Given the inconsistent results from these studies, the present meta-analysis aimed to obtain a more precise estimate of the association between $I L-21$ rs907715 and rs 2221903 polymorphisms and SLE. Studies regarding these specific polymorphisms and SLE were retrieved from PubMed, Embase, Web of Science, CNKI, and CBM. Data were extracted and meta-analysis was performed using the STATA 12.0 software. For the $I L-21$ rs907715 polymorphism, seven sets of comparisons involving 7977 SLE cases and 8097 healthy controls were considered. Results showed that there were significant differences in the $I L-21$ rs907715 genotype distribution between SLE patients and
\end{abstract}


healthy controls in the comparisons of all genetic models. Upon stratified analysis by ethnicity, a similar result was found in the Caucasian and African-American population. For the $I L-21$ rs2221903 polymorphism, seven sets of comparisons involving 7990 SLE cases and 8098 healthy controls were considered. Results showed that there were significant differences in the $I L-21$ rs2221903 genotype distribution between SLE patients and healthy controls in the comparisons of GG versus AA and GG versus GA+AA. Upon stratified analysis by ethnicity, a similar result was found in the Caucasian population. This meta-analysis suggests that the both $I L-21$ rs 907715 and rs2221903 polymorphisms may be associated with SLE susceptibility. As current evidence remains limited, further studies are needed to warrant the association between IL-21 rs907715 and rs2221903 polymorphisms and SLE susceptibility.

Key words: Interleukin 21; Polymorphism; Meta-analysis; Systemic lupus erythematosus

\section{INTRODUCTION}

Systemic lupus erythematosus (SLE) is a chronic autoimmune disease characterized by the production of a range of autoantibodies (Tsokos, 2011). The exact etiology and pathogenesis of SLE has not been clarified thus far, however there is evidence that B and T cells are critical to the development of SLE (Sarra and Monteleone, 2010). Interleukin-21 (IL-21) is recognized as a member of the type I cytokine family, mainly synthesized by a range of differentiated $\mathrm{CD}^{+}{ }^{+} \mathrm{T}$ helper (Th) cells (Monteleone et al., 2009) and activated natural killer T (NKT) cells (Coquet et al., 2007). Studies have shown that IL-21 has an important role in the control of the growth, survival, differentiation, and function of both B (Mehta et al., 2003; Ozaki et al., 2004; Jin et al., 2004; Konforte et al., 2009) and T cells (Strengell et al., 2002; Fröhlich et al., 2007; Monteleone et al., 2008). There is increasing evidence that IL-21 contributes to the pathogenesis of SLE. For instance, elevated serum and mRNA levels of IL-21 has been detected in BXSB-Yaa mice, a mouse model which develops severe SLE-related symptoms, including hypergammaglobulinemia, autoantibody production, reduced frequencies of marginal zone B cells and monocytosis, and renal disease (Bubier et al., 2009). Conversely, IL-21 receptor (IL-21R)-deficient mice exhibited none of these abnormalities (Bubier et al., 2009). One study in humans reported that serum levels of IL-21 was markedly increased in SLE patients compared with healthy controls (Wong et al., 2010).

Although possible associations of the $I L-21$ polymorphisms with SLE were reported, and many case-control studies were further performed to identify the association between IL-21 rs907715 and rs2221903 polymorphisms and SLE (Sawalha et al., 2008; Hughes et al., 2011; Leng et al., 2012; Ding et al., 2012; Lan et al., 2014). However, results from these studies were inconsistent, which may be due to the limitations in sample size and differences in ethnic populations. Therefore, we performed a meta-analysis to evaluate comprehensively the association between the $I L-21$ rs 907715 and rs2221903 polymorphisms and SLE based on all eligible case-control studies. 


\section{MATERIAL AND METHODS}

\section{Literature search}

The studies regarding the association between $I L-21$ rs907715 and rs2221903 polymorphisms and SLE published up to October 2014 without language restrictions were independently searched by two authors in the PubMed, Embase, Web of Science, Chinese National Knowledge Infrastructure (CNKI) and Chinese Biomedical (CBM) Literature Database, using the following terms: ("Interleukin-21" or "IL-21") and ("Systemic lupus erythematosus" or "SLE") and ("polymorphism" or "SNP" or "single nucleotide polymorphism" or "variation" or "mutation"). The bibliographies of retrieved articles were manually searched to find additional relevant studies.

\section{Study selection}

Studies were included in this meta-analysis if they met the following criteria: (a) casecontrol studies focused on associations between $I L-21$ rs 907715 or rs2221903 polymorphisms and SLE, (b) 95\% confidence interval (CI) for odds ratio (OR) were available or could be calculated, and (c) the distribution of genotypes in the control group was consistent with HardyWeinberg equilibrium (HWE). When met with repetitive publications, only one publication was included. Family-based studies were also excluded.

\section{Data extraction}

The following data from the studies included were extracted independently by two authors, including the first author, year of publication, country of subject recruitment, subjects' ethnicities, sample size, and genotype distributions in SLE cases and controls. In case of conflicting evaluations, disagreements were resolved through discussions between the authors.

\section{Statistical analysis}

Genotype distributions in the controls were tested for HWE using the Pearson's $\chi^{2}$ test (Schaid and Jacobsen, 1999). Between-study heterogeneity was checked by the Cochran Q-statistic and $\mathrm{I}^{2}$ test (Higgins and Thompson, 2002; Zintzaras and Ioannidis, 2005). When P $<0.1$ for Q-test or $\mathrm{I}^{2}>50 \%$ indicated the existence of heterogeneity, a random-effect model was used; otherwise, a fixed-effect model was applied. ORs with corresponding 95\% confidence intervals (CIs) were calculated to assess the association between $I L-21$ rs907715 and rs2221903 polymorphisms and SLE under four genetic models: G allele versus A allele, GG versus $\mathrm{AA}, \mathrm{GG}+\mathrm{GA}$ versus $\mathrm{AA}$, and $\mathrm{GG}$ versus $\mathrm{GA}+\mathrm{AA}$. The significance of the pooled $\mathrm{OR}$ was determined using the Z-test. To evaluate whether the association showed any ethnicity specific effects, we analyzed the data for separate subgroups defined by ethnicity. Sensitivity analysis was performed by sequential omission of individual studies and recalculating the results in order to assess the stability of the results. Begg's funnel plots and Egger's test were used to investigate whether publication bias might affect the validity of the estimates (Peters et al., 2006). All the statistical tests were conducted using the STATA 12.0 software. 


\section{RESULTS}

\section{Characteristics of the studies included}

The flow chart of the selection of studies and specific reasons for exclusion from the meta-analysis are shown in Figure 1. The search strategy retrieved twenty-six potentially relevant studies. In accordance with the inclusion criteria, five articles were included in this meta-analysis and all of them were written in English. The publication year of the articles included ranged from 2008 to 2014 . For the $I L-21$ rs 907715 polymorphism, seven sets of comparisons involving 7977 SLE cases and 8097 healthy controls were considered. For the IL-21 rs2221903 polymorphism, seven sets of comparisons involving 7990 SLE cases and 8098 healthy controls were considered. Among the eligible seven sets of comparisons, three comparisons were conducted in the Asian population, two comparisons were conducted in the Caucasian population, and the remaining two comparisons were conducted in the African-American population. The distribution of genotypes within the control group of each comparison was consistent with HWE (all P > 0.05). The control group was chosen from healthy individuals without any systemic and dermatologic diseases. The characteristics of the included studies were summarized in Table 1.

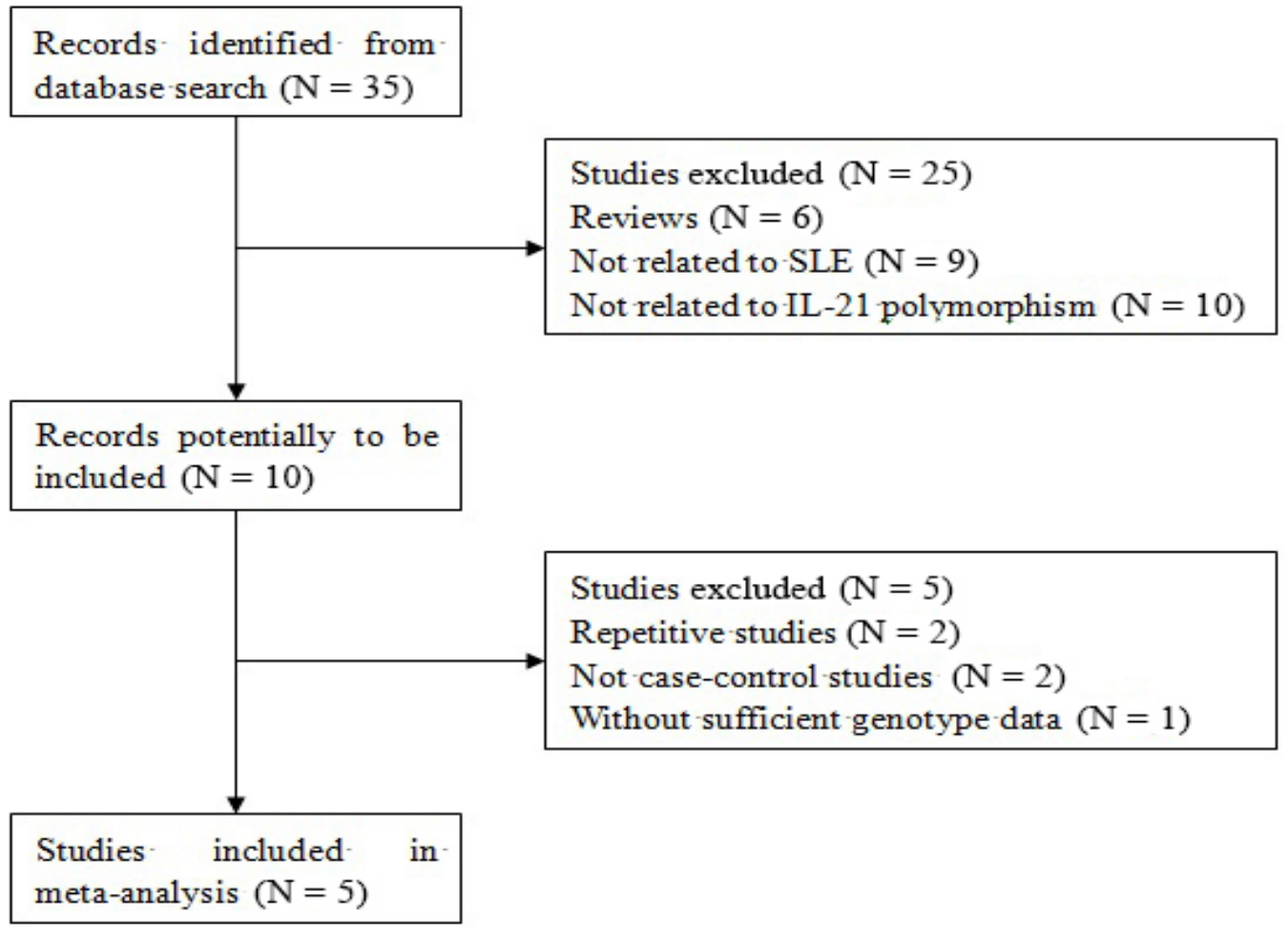

Figure 1. Selection of studies and specific reasons for exclusion from the meta-analysis. 


\begin{tabular}{|c|c|c|c|c|c|c|c|c|c|c|c|}
\hline \multirow[t]{3}{*}{ Author } & \multirow[t]{3}{*}{ Year } & \multirow[t]{3}{*}{ Country } & \multirow[t]{3}{*}{ Ethnic } & \multicolumn{2}{|c|}{ Number } & \multicolumn{6}{|c|}{ Genotype } \\
\hline & & & & \multirow[t]{2}{*}{ Case } & \multirow[t]{2}{*}{ Controls } & \multicolumn{3}{|c|}{ Case } & \multicolumn{3}{|c|}{ Control } \\
\hline & & & & & & $\mathrm{AA}$ & $\mathrm{AG}$ & $\overline{\mathrm{GG}}$ & $\mathrm{AA}$ & $\mathrm{AG}$ & GG \\
\hline \multicolumn{12}{|l|}{ rs907715 } \\
\hline Sawalha et al. (2008) & 2008 & USA & Caucasian & 644 & 644 & 67 & 281 & 296 & 93 & 304 & 247 \\
\hline Sawalha et al. (2008) & 2008 & USA & African-American & 366 & 366 & 49 & 170 & 147 & 61 & 171 & 134 \\
\hline Hughes et al. (2011) & 2011 & USA & Caucasian & 3918 & 3503 & 387 & 1688 & 1843 & 417 & 1582 & 1504 \\
\hline Hughes et al. (2011) & 2011 & USA & African-American & 1411 & 1761 & 199 & 662 & 550 & 294 & 851 & 616 \\
\hline Ding et al. (2012) & 2012 & China & Asian & 605 & 666 & 124 & 293 & 188 & 128 & 339 & 199 \\
\hline Leng et al. (2012) & 2012 & China & Asian & 858 & 967 & 151 & 418 & 289 & 207 & 481 & 279 \\
\hline Lan et al. (2014) & 2014 & China & Asian & 175 & 190 & 41 & 93 & 41 & 35 & 97 & 58 \\
\hline \multicolumn{12}{|l|}{ rs 2221903} \\
\hline Sawalha et al. (2008) & 2008 & USA & Caucasian & 644 & 644 & 298 & 280 & 66 & 324 & 275 & 45 \\
\hline Sawalha et al. (2008) & 2008 & USA & African-American & 366 & 366 & 303 & 57 & 6 & 324 & 40 & 2 \\
\hline Hughes et al. (2011) & 2011 & USA & Caucasian & 3928 & 3499 & 1844 & 1694 & 390 & 1740 & 1455 & 304 \\
\hline Hughes et al. (2011) & 2011 & USA & African-American & 1414 & 1766 & 1222 & 185 & 7 & 1509 & 247 & 10 \\
\hline Ding et al. (2012) & 2012 & China & Asian & 605 & 666 & 483 & 116 & 6 & 495 & 166 & 5 \\
\hline Leng et al. (2012) & 2012 & China & Asian & 858 & 967 & 672 & 175 & 11 & 787 & 171 & 9 \\
\hline Lan et al. (2014) & 2014 & China & Asian & 175 & 190 & 122 & 50 & 3 & 143 & 45 & 2 \\
\hline
\end{tabular}

\section{Meta-analysis results}

For the $I L-21$ rs 907715 polymorphism, significant heterogeneity between studies was observed in the comparisons of $\mathrm{G}$ allele versus A allele, and GG versus AA with the Q test and the $\mathrm{I}^{2}$ test $\left(\mathrm{P}<0.1\right.$ or $\left.\mathrm{I}^{2}>50 \%\right)$. Therefore, the random effects model was used to pool the results. No heterogeneity between studies was observed in the comparisons of GG+GA versus AA, and GG versus GA+AA and the fixed effects model was used to pool the results. Metaanalysis results showed that there were significant differences in the $I L-21$ rs 907715 genotype distribution between SLE patients and healthy controls in the comparisons of $G$ allele versus A allele, $\mathrm{GG}$ versus $\mathrm{AA}, \mathrm{GG}+\mathrm{GA}$ versus $\mathrm{AA}$, and $\mathrm{GG}$ versus $\mathrm{GA}+\mathrm{AA}$ (respectively: $\mathrm{OR}=1.13$, $95 \% \mathrm{CI}=1.05-1.21, \mathrm{P}=0.001 ; \mathrm{OR}=1.27,95 \% \mathrm{CI}=1.09-1.48, \mathrm{P}=0.003 ; \mathrm{OR}=1.20,95 \% \mathrm{CI}$ $=1.09-1.31, \mathrm{P}=0.000 ; \mathrm{OR}=1.18,95 \% \mathrm{CI}=1.11-1.26, \mathrm{P}=0.000)$. Upon stratified analysis by ethnicity, a similar result was found in Caucasian and African-American populations. Results for the $I L-21$ rs 907715 polymorphism were summarized in Table 2. For the $I L-21$ rs 2221903 polymorphism, significant heterogeneity between studies was observed in the comparisons of $\mathrm{G}$ allele versus $\mathrm{A}$ allele, and $\mathrm{GG}+\mathrm{GA}$ versus $\mathrm{AA}$, and the random effects model was used to pool the results. No heterogeneity between studies was observed in the comparisons of GG versus AA, and GG versus GA+AA, and the fixed effects model was used to pool the results. Meta-analysis results showed that there were significant differences in the $I L-21 \mathrm{rs} 2221903$ genotype distribution between SLE patients and healthy controls in the comparisons of GG versus AA, and GG versus GA+AA. Upon stratified analysis by ethnicity, a similar result was found in the Caucasian population. Results for the $I L-21$ rs2221903 polymorphism were summarized in Table 3.

\section{Sensitivity analysis and publication bias}

Sensitivity analyses were performed by sequential omission of individual studies for 
all subjects and subgroups. The pooled ORs were not significantly altered in all subjects and subgroups by omitting any single study (data not shown). The results of the sensitivity analyses indicated the stability of our results. Begg's funnel plot and Egger's test showed that there were no statistically significant publication biases in all genetic models (all $\mathrm{P}>0.05$ ) (data not shown).

Table 2. Meta-analysis of the association between IL-21 rs907715 polymorphism and SLE susceptibility.

\begin{tabular}{|c|c|c|c|c|c|c|}
\hline Genetic model & Population & OR $(95 \% \mathrm{CI})$ & $\mathrm{P}$ & Statistical model & $\mathrm{I}^{2}$ & $\mathrm{P}_{\text {Heterogeneity }}$ \\
\hline \multirow[t]{4}{*}{$\mathrm{G} v s \mathrm{~A}$} & Overall & $1.13(1.05-1.21)$ & 0.001 & Random & $49.6 \%$ & 0.064 \\
\hline & Caucasian & $1.17(1.10-1.25)$ & 0.000 & Fixed & $41.1 \%$ & 0.192 \\
\hline & African-American & $1.15(1.05-1.26)$ & 0.003 & Fixed & $0.0 \%$ & 0.958 \\
\hline & Asian & $1.01(0.82-1.24)$ & 0.925 & Random & $74.1 \%$ & 0.021 \\
\hline \multirow[t]{4}{*}{ GG $v s \mathrm{AA}$} & Overall & $1.27(1.09-1.48)$ & 0.003 & Random & $48.8 \%$ & 0.068 \\
\hline & Caucasian & $1.37(1.19-1.58)$ & 0.000 & Fixed & $26.3 \%$ & 0.244 \\
\hline & African-American & $1.33(1.10-1.61)$ & 0.004 & Fixed & $0.0 \%$ & 0.890 \\
\hline & Asian & $1.01(0.66-1.53)$ & 0.969 & Random & $74.4 \%$ & 0.020 \\
\hline \multirow[t]{4}{*}{$\mathrm{GG}+\mathrm{GA} v s \mathrm{AA}$} & Overall & $1.20(1.09-1.31)$ & 0.000 & Fixed & $32.0 \%$ & 0.184 \\
\hline & Caucasian & $1.27(1.11-1.45)$ & 0.001 & Fixed & $0.0 \%$ & 0.378 \\
\hline & African-American & $1.23(1.03-1.47)$ & 0.019 & Fixed & $0.0 \%$ & 0.800 \\
\hline & Asian & $1.01(0.75-1.36)$ & 0.972 & Random & $62.5 \%$ & 0.069 \\
\hline \multirow[t]{4}{*}{$\mathrm{GG} v s \mathrm{GA}+\mathrm{AA}$} & Overall & $1.18(1.11-1.26)$ & 0.000 & Fixed & $22.7 \%$ & 0.256 \\
\hline & Caucasian & $1.21(1.11-1.31)$ & 0.000 & Fixed & $30.3 \%$ & 0.231 \\
\hline & African-American & $1.18(1.04-1.35)$ & 0.012 & Fixed & $0.0 \%$ & 0.899 \\
\hline & Asian & $1.04(0.80-1.36)$ & 0.751 & Random & 63.0 & 0.067 \\
\hline
\end{tabular}

$\mathrm{OR}=$ odds ratio $\mathrm{CI}=$ confidence interval. Bold values mean that their association is significant.

Table 3. Meta-analysis of the association between IL-21 rs2221903 polymorphism and SLE susceptibility.

\begin{tabular}{|c|c|c|c|c|c|c|}
\hline Genetic model & Population & OR $(95 \% \mathrm{CI})$ & $\mathrm{P}$ & Statistical model & $\mathrm{I}^{2}$ & $\mathrm{P}_{\text {Heterogeneity }}$ \\
\hline \multirow[t]{4}{*}{$\mathrm{G} v s \mathrm{~A}$} & Overall & $1.09(0.96-1.24)$ & 0.172 & Random & $64.3 \%$ & 0.010 \\
\hline & Caucasian & $1.11(1.04-1.19)$ & 0.001 & Fixed & $0.0 \%$ & 0.400 \\
\hline & African-American & $1.19(0.69-2.07)$ & 0.527 & Random & $84.4 \%$ & 0.011 \\
\hline & Asian & $1.04(0.76-1.44)$ & 0.796 & Random & $75.8 \%$ & 0.016 \\
\hline \multirow[t]{4}{*}{ GG $v s \mathrm{AA}$} & Overall & $1.27(1.09-1.48)$ & 0.002 & Fixed & $0.0 \%$ & 0.732 \\
\hline & Caucasian & $1.26(1.08-1.46)$ & 0.003 & Fixed & $33.1 \%$ & 0.221 \\
\hline & African-American & $1.28(0.58-2.82)$ & 0.543 & Fixed & $46.9 \%$ & 0.170 \\
\hline & Asian & $1.40(0.72-2.72)$ & 0.314 & Fixed & $0.0 \%$ & 0.947 \\
\hline \multirow{4}{*}{$\mathrm{GG}+\mathrm{GA} v s \mathrm{AA}$} & Overall & $1.09(0.94-1.25)$ & 0.265 & Random & $64.5 \%$ & 0.010 \\
\hline & Caucasian & $1.13(1.04-1.23)$ & 0.006 & Fixed & $0.0 \%$ & 0.678 \\
\hline & African-American & $1.18(0.69-2.02)$ & 0.551 & Random & $81.5 \%$ & 0.020 \\
\hline & Asian & $1.03(0.71-1.51)$ & 0.865 & Random & $78.8 \%$ & 0.009 \\
\hline \multirow[t]{4}{*}{$\mathrm{GG} v s \mathrm{GA}+\mathrm{AA}$} & Overall & $1.28(1.12-1.48)$ & 0.000 & Fixed & $0.0 \%$ & 0.811 \\
\hline & Caucasian & $1.28(1.11-1.48)$ & 0.001 & Fixed & $0.0 \%$ & 0.339 \\
\hline & African-American & $1.25(0.57-2.76)$ & 0.577 & Fixed & $48.2 \%$ & 0.165 \\
\hline & Asian & $1.37(0.71-2.66)$ & 0.349 & Fixed & 0.0 & 0.958 \\
\hline
\end{tabular}

$\mathrm{OR}=$ odds ratio; $\mathrm{CI}=$ confidence interval. Bold values mean that their association is significant.

\section{DISCUSSION}

In humans, the $I L-21$ gene is located on chromosome 4q26-q27, which consists of 5 exons spanning approximately $8.44 \mathrm{~kb}$ of genomic DNA. Several polymorphisms in the $I L-21$ gene have been identified and several studies have been carried out to identify whether $I L-21$ polymorphisms were associated with SLE susceptibility (Sawalha et al., 2008; Hughes et al., 2011; Leng et al., 2012; Ding et al., 2012; Lan et al., 2014). Most of the studies focused on $I L$ 21 rs907715 and rs2221903 polymorphisms. However, the results from these studies were in- 
consistent, which may be partly due to a small sample size in individual studies or differences in various ethnic groups. Meta-analysis has been recognized as a useful statistical method that combines findings from independent studies to precisely evaluate the effect of selected genetic polymorphisms on the risk of disease (Attia et al., 2003). To the best of our knowledge, no meta-analysis has been conducted to evaluate the association between $I L-21$ polymorphisms and SLE susceptibility. We performed the present meta-analysis based on all eligible casecontrol studies, to provide a more complete picture of the role of $I L-21$ polymorphisms in SLE susceptibility, as compared with that published in individual studies.

In this meta-analysis, all the studies checked genotypes for quality control. The genotype distribution of controls in all studies was consistent with HWE. In the meta-analysis of the rs 907715 polymorphism, seven sets of comparisons involving 7977 SLE cases and 8097 healthy controls were considered. When all the eligible studies were pooled into the metaanalysis, the results showed that $I L-21$ rs 907715 polymorphism was associated with SLE susceptibility under all genetic models. Sensitivity analysis also showed that omission of any single study did not have a significant impact on the combined ORs. Furthermore, a funnel plot did not reveal obvious asymmetry, and the Egger test further indicated no considerable publication bias in this meta-analysis. This made the results of this meta-study more reliable to some extent. To evaluate whether the association showed any ethnicity specific effects, we analyzed the data for separate subgroups defined by ethnicity. Upon stratified analysis, a similar result was found in Caucasian and African-American populations. Moreover, no heterogeneity between studies was observed in Caucasian and African-American subgroups. For the $I L-21$ rs2221903 polymorphism, seven sets of comparisons involving 7990 SLE cases and 8098 healthy controls were considered. Meta-analysis results showed that there were significant differences in the $I L-21$ rs2221903 genotype distribution between SLE patients and healthy controls in the comparisons of GG versus AA, and GG versus GA+AA. Upon stratified analysis by ethnicity, a similar result was found in the Caucasian population.

Some limitations of our meta-analysis should be acknowledged. First, the completeness of evidence may be impeded by publication bias, language bias (Juni et al., 2002) and inadequate reporting (Little et al., 2009), despite the comprehensive study identification process and lack of overt publication bias in the present study. Second, the number of studies included was not sufficiently large, especially in ethnicity subgroup analysis, which may not provide enough statistical power to explore the real association between $I L-21$ rs2221903 polymorphisms and SLE susceptibility. Third, SLE is polygenic and may also be modulated by several other genetic markers beyond $I L-21$, including $I L-10$ (Liu et al., 2013), MCP-I (Zhou et al., 2014a), CTLA-4 (Zhu et al., 2014), the vitamin D receptor (Zhou et al., 2014b), and several other candidate genes. Thus, our meta-analysis emphasizes that elucidating the pathogenesis of SLE would demand further evaluation of the potential gene-gene interactions. Finally, due to the lack of raw data, we have not considered some factors when analyzing the data, such as the factors of gender, age and the severity of the disease, which may cause serious confounding bias. However, the advantages of this meta-analysis were also obvious. First, compared with individual studies, the sample size of our study was larger, which made the results more reliable. Second the associations between $I L-21$ rs907715 polymorphisms and SLE were evaluated under different genetic models.

In summary, our meta-analysis suggests that the $I L-21$ rs 2221903 polymorphism may be associated with SLE, especially in the Caucasian population. As few studies are available 
in this field and current evidence remains limited, well-designed and large studies are needed to investigate further the association between $I L-21$ rs2221903 polymorphisms and SLE.

\section{Conflicts of interest}

The authors declare no conflict of interest.

\section{REFERENCES}

Attia J, Thakkinstian A and D'Este C (2003). Meta-analyses of molecular association studies: methodologic lessons for genetic epidemiology. J. Clin. Epidemiol. 56: 297-303.

Bubier JA, Sproule TJ, Foreman O, Spolski R, et al. (2009). A critical role for IL-21 receptor signaling in the pathogenesis of systemic lupus erythematosus in BXSB-Yaa mice. Proc. Natl. Acad. Sci. U. S. A. 106: 1518-1523.

Coquet JM, Kyparissoudis K, Pellicci DG, Besra G, et al. (2007). IL-21 is produced by NKT cells and modulates NKT cell activation and cytokine production. J. Immunol. 178: 2827-2834.

Ding L, Wang S, Chen GM, Leng RX, et al. (2012). A single nucleotide polymorphism of IL-21 gene is associated with systemic lupus erythematosus in a Chinese population. Inflammation 35: 1781-1785.

Fröhlich A, Marsland BJ, Sonderegger I, Kurrer M, et al. (2007). IL-21 receptor signaling is integral to the development of Th2 effector responses in vivo. Blood 109: 2023-2031.

Higgins JP and Thompson SG (2002). Quantifying heterogeneity in a meta-analysis. Stat. Med. 21: 1539-1558.

Hughes T, Kim-Howard X, Kelly JA, Kaufman KM, et al. (2011). Fine-mapping and transethnic genotyping establish IL2/ IL21 genetic association with lupus and localize this genetic effect to IL21. Arthritis Rheum. 63: 1689-1697.

Jin H, Carrio R, Yu A and Malek TR (2004). Distinct activation signals determine whether IL-21 induces B cell costimulation, growth arrest, or Bim-dependent apoptosis. J. Immunol. 173: 657-665.

Juni P, Holenstein F, Sterne J, Bartlett C, et al. (2002). Direction and impact of language bias in meta-analyses of controlled trials: empirical study. Int. J. Epidemiol. 31: 115-123.

Konforte D, Simard N and Paige CJ (2009). IL-21: an executor of B cell fate. J. Immunol. 182: 1781-1787.

Lan Y, Luo B, Wang JL, Jiang YW, et al. (2014). The association of interleukin-21 polymorphisms with interleukin-21 serum levels and risk of systemic lupus erythematosus. Gene 538: 94-98.

Leng RX, Wang W, Cen H, Zhou M, et al. (2012). Gene-gene and gene-sex epistatic interactions of MiR146a, IRF5, IKZF1, ETS1 and IL21 in systemic lupus erythematosus. PLoS One 7: e51090.

Little J, Higgins JP, Ioannidis JP, Moher D, et al. (2009). Strengthening the reporting of genetic association studies (STREGA): an extension of the strengthening the reporting of observational studies in epidemiology (STROBE) statement. J. Clin. Epidemiol. 62: 597-608 e594.

Liu P, Song J, Su H, Li L, et al. (2013). IL-10 gene polymorphisms and susceptibility to systemic lupus erythematosus: a meta-analysis. PLoS One 8: e69547.

Mehta DS, Wurster AL, Whitters MJ, Young DA, et al. (2003). IL-21 induces the apoptosis of resting and activated primary B cells. J. Immunol. 170: 4111-4118.

Monteleone G, Pallone F and MacDonald TT (2008). Interleukin-21: a critical regulator of the balance between effector and regulatory T-cell responses. Trends Immunol. 29: 290-294.

Monteleone G, Pallone F and Macdonald TT (2009). Interleukin-21 as a new therapeutic target for immune-mediated diseases. Trends Pharmacol. Sci. 30: 441-447.

Ozaki K, Spolski R, Ettinger R, Kim HP, et al. (2004). Regulation of B cell differentiation and plasma cell generation by IL-21, a novel inducer of Blimp-1 and Bcl-6. J. Immunol. 173: 5361-5371.

Peters JL, Sutton AJ, Jones DR, Abrams KR, et al. (2006). Comparison of two methods to detect publication bias in metaanalysis. JAMA 295: 676-680.

Sarra M and Monteleone G (2010). Interleukin-21: a new mediator of inflammation in systemic lupus erythematosus. $J$. Biomed. Biotechnol. 2010: 294582.

Sawalha AH, Kaufman KM, Kelly JA, Adler AJ, et al. (2008). Genetic association of interleukin-21 polymorphisms with systemic lupus erythematosus. Ann. Rheum. Dis. 67: 458-461.

Schaid DJ and Jacobsen SJ (1999). Biased tests of association: comparisons of allele frequencies when departing from Hardy-Weinberg proportions. Am. J. Epidemiol. 149: 706-711.

Strengell M, Sareneva T, Foster D, Julkunen I, et al. (2002). IL-21 up-regulates the expression of genes associated with innate immunity and Th1 response. J. Immunol. 169: 3600-3605.

Tsokos GC (2011). Systemic lupus erythematosus. N. Engl. J. Med. 365: 2110-2121.

Genetics and Molecular Research 14 (3): 9595-9603 (2015)

CFUNPEC-RP www.funpecrp.com.br 
Wong CK, Wong PT, Tam LS, Li EK, et al. (2010). Elevated production of B cell chemokine CXCL13 is correlated with systemic lupus erythematosus disease activity. J. Clin. Immunol. 30: 45-52.

Zhou TB, Jiang ZP, Liang MJ and Huang YJ (2014a). Relationship between MCP-1 promoter -2518 A/G gene polymorphism (rs1024611) and systemic lupus erythematosus/lupus nephritis. J. Recept. Signal Transduct. Res. 1-9.

Zhou TB, Jiang ZP, Lin ZJ and Su N (2014b). Association of vitamin D receptor gene polymorphism with the risk of systemic lupus erythematosus. J. Recept. Signal Transduct. Res. 22: 1-7.

Zhu Y, Wang J and Feng X (2014). CTLA-4 SNPs (CT60A/G, -1722T/C, -1661G/A, and -318C/T) and systemic lupus erythematosus: a meta-analysis. Crit. Rev. Eukaryot. Gene Exp. 24: 89-100.

Zintzaras E and Ioannidis JP (2005). Heterogeneity testing in meta-analysis of genome searches. Genet. Epidemiol. 28 : 123-137. 